\title{
Commercialising Size Survey Data SizeUK - The UK National Size Survey
}

\author{
Andrew Crawford ${ }^{\mathrm{a}}$ and Alexandre Kung ${ }^{\mathrm{b}}$ \\ âSizemic Ltd., London, UK \\ bINSITUT TPC, Hong Kong, China
}

\begin{abstract}
Size surveys and 3D body data, derived from 3D body scanning, provides a wealth of measurement and shape data; potentially of enormous value to the Retail \& Fashion, Healthcare and other sectors. The challenge is to deliver the data in a form enabling retailers and clothing companies to utilise the results and realise the commercial benefits, so extending the value of the survey beyond purely an academic research project. This paper therefore seeks to demonstrate how Size Survey data, specifically SizeUK, can be utilised and exploited through a portfolio of products and services, an endto-end solution, taking retailers from acquisition of the data, through data analysis and size chart development, to practical tools like customised fit mannequins and 3D pattern development. The objective being to help them understand the distribution and overall size and shape profile of their target customers, to improve the sizing and fit of their garments and maximise the percentage of their target customer that can fit their clothes.
\end{abstract}

This paper also seeks to demonstrate the importance and added value of 3D shape data in terms of implementing the results and maximising the benefits.

Keywords: 3D Body scanning, size survey, 3D shape analysis, fit mannequins, 3D pattern generation

\section{Introduction}

Sizemic is a fashion technology company, based in London, providing a 'one stop' sizing and fit solution for the fashion industry. We specialise in products and services based on 3D body data derived from 3D body scanning - from size survey data to size chart development; fit mannequins to 3D pattern development. Sizemic is responsible for hosting the SizeUK data and has the exclusive rights to sell the data on behalf of the SizeUK consortium.

Our technology partner, INSTITUT TPC is a garment technology consulting firm, based in Hong Kong, which has pioneered an innovative and scientific approach to the development of garment patterns in a 3D environment.

Size surveys and 3D body data, derived from 3D body scanning, provides a wealth of measurement and shape data; potentially of enormous value to the Retail \& Fashion, Healthcare and other sectors. The problem is that users, particularly Retailers, are confronted with a vast amount of data. The challenge is to deliver the data in a form that enables retailers and clothing companies to utilise the results and realise the commercial benefits. In so doing this extends the value of the survey beyond what would otherwise be a purely academic research project. This paper therefore seeks to demonstrate how Size Survey data, specifically SizeUK, can be utilised and exploited through a portfolio of products and services, an end-to-end solution, taking retailers from acquisition of the data, through data analysis and size chart development, to practical tools like customised fit mannequins and $3 \mathrm{D}$ pattern development. The objective being to help them understand the distribution and overall size and shape profile of their target customers, to improve the sizing and fit of their garments and maximise the percentage of their target customer that can fit their clothes.

Given that 3D shape is more important to achieving good fit than linear measurements measurements do not describe shape and people with similar measurements can have very different shape profiles - this paper also seeks to demonstrate the importance and added value of 3D shape data in terms of implementing the results and maximising the benefits.

\footnotetext{
a andrew.crawford@sizemic.eu +44 (0)20 30512644 www.sizemic.eu

b akung@tpc.com.hk +852 27218716 www.tpc-intl.com
} 
The paper is divided into 4 main sections:

- Overview of SizeUK - The UK National Size Survey

- Measurement Analysis, 3D Shape Analysis \& Size Chart Development

- Customised and Generic fit Mannequins

- 3D Pattern Development

\section{SizeUK - The UK National Size Survey}

SizeUK - the UK National Sizing Survey is a survey of 11,000 subjects using 3D whole body scanners to automatically extract over 150 body measurements from each subject. This highly accurate size and shape data, together with each subject's 3D point cloud, is held in an anthropometric database, available for online data mining. SizeUK is a collaboration of the UK Government, 17 major UK retailers, leading academics and technology companies. The retailers were responsible for recruiting subjects to be measured, with subjects being encouraged to register online.

The principal UK clothing colleges managed the data collection process; seen as an educational opportunity. The USA scanner company [TC]2 developed special size extraction software to automatically extract over 150 'SizeUK' measurements from each body scan, reducing manual measuring to just 8 measurements, such as height and weight. Initial data collection took 6 months and was completed in 2002, the headline results were announced in 2004. SizeUK is the first national size survey in the UK since the 1950's. Sizemic is hosting the data on a secure, web-database service accessible through proprietary online data analysis tools and has the exclusive rights to sell the data on behalf of the Retailers. The potential commercial benefits for Retail clothing brands acquiring the data are considerable.

The original SizeUK Retail Partners are Arcadia Group, BHS, Debenhams, Otto UK, Great Universal Stores (now Shop Direct), House of Fraser, John Lewis Partnership, Littlewoods Retail, Marks \& Spencer, N Brown Group, Monsoon Accessorize, Oasis Stores, Redcats UK, Rohan Designs, Speedo International, Tesco Stores.

\subsection{Recruitment \& Data Collection}

To be statistically reliable and credible, a size survey needs to meet a number of key criteria with respect to sampling and data collection:

- The sample needs to be large enough to be a statistically representative sample of the population.

- The sample needs to be representative of the population in respect of key demographic criteria - gender, age, ethnicity, socio-economic groups etc.

- To be a true National survey the data needs to be collected from the main geographical regions of the country.

- The data also needs to be collected over a relatively short period of time, e.g. 6 months, for it to be a true 'snapshot' of the nation at a given point in time.

Volunteers for SizeUK were recruited through publicity in the national media, the retailers and the SizeUK web site. Subjects were then selected for the survey using quota sampling to meet the national statistics of age, ethnic origin, socio economic group and geo-demographics. The aim was to measure a statistically representative sample of the UK population of approximately 5,000 females and 5,000 male subjects between the ages of 16 and 85+. 5,800 females and 5,300 were actually scanned. Children were not included in the survey but a separate children's survey is being planned. There were 8 data collection points covering 3 geographic regions (Scotland/North of England, the Midlands and the South):

$\begin{array}{llll}\text { Southampton } & \text { Cardiff } & \text { Birmingham } & \text { Manchester } \\ \text { London } & \text { Nottingham } & \text { Leeds } & \text { Edinburgh }\end{array}$

Subjects, wearing underwear, were scanned in 2 poses, standing and seated. 8-10 manual measurements, not covered by the scanning process were also taken. Each subject was asked to complete an extensive market research questionnaire which captured information on personal details, clothing issues, shopping habits and preferences, lifestyle habits including health \& fitness, occupation and employment and ethnicity. Measuring took place over a 6 month period between August 2001 and March 2002. 


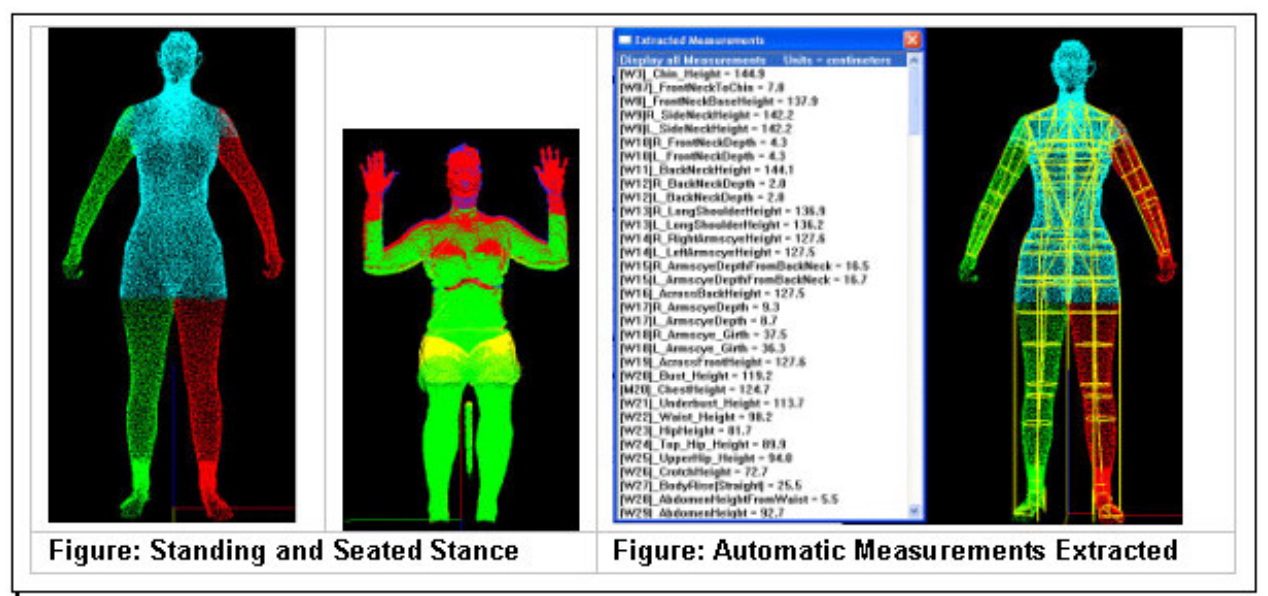

Fig 1. SizeUK - Standing and seated scanning poses and automatic measurement extraction

\subsection{Size UK Database}

Information in the database includes:

- Personal details on each subject (excluding name \& addresses)

- 11,000 subjects - approximately $50 \%$ women and $50 \%$ men

- 22,000 3D body scans - 2 per subject (one standing, one seated)

- Over 150 measurements per subject

- Market Research Information - results of 40 questions per subject on clothes, shopping and lifestyle habits.

\subsection{Survey Results}

The results of SizeUK are available to licence holders through:

- Standard Data Analysis of the measurements, shape and market research data:

$>$ Statistical analysis of the measurement information covering mean, max/min standard deviation etc in total and by age, region, socio economic group or ethnicity.

$>$ Frequency distribution charts and analysis for each measurement

3D shape analysis of the scan data

> Cross-correlation charts showing the relationship between key measurements.

> Tabulated statistical analysis of the Market Research Information.

- Customised Data Analysis through online access to the SizeUK database using a set of software tools designed by University College London (UCL). This allows users to conduct their own analysis on a subset of the data based on their customer profile.

- Data Analysis Services provided by Sizemic. Sizemic offers a range of basic and advanced customised data analysis services for customers who would like analysis carried out on their behalf.

\section{Commercialising the Data}

Whilst SizeUK provides a rich and comprehensive database of measurement and shape data it does not provide users with standard size charts based on UK garment size designations. For several reasons this was not an objective of the project. Definitions of UK size designations vary in practice, as do the increments between sizes and different types of size designation are used. It was also expected that the Retailers would want to create new size charts for their specific customer profile, rather than size charts based on broad national averages. Retailers were therefore faced with a vast amount of data and a relatively complex task. The challenge is to deliver the data in a form enabling retailers and clothing companies to utilise the results and realise the commercial benefits, so extending the value of the survey beyond purely an academic research project. 
Sizemic recognised the need to provide customised data analysis services and the requirement for a range of related products and services to enable them to implement the results and maximise the commercial benefits of investing in the SizeUK data. Sizemic developed a portfolio of products and services, an end-to-end solution, taking retailers from acquisition of the data, through data analysis and size chart development, to practical tools like customised fit mannequins and 3D pattern development. The objective being to help them understand the distribution and overall size and shape profile of their target customers, to improve the sizing and fit of their garments and maximise the percentage of their target customer that can fit their clothes.

A recurrent theme is the importance and value of $3 D$ shape data and the principle that the process is driven more by shape than linear measurements.

\subsection{Measurement Analysis}

The process starts with a programme of customised data analysis on a subset of the SizeUK data based on the retailer's target customer profile. This can be a range of criteria but typically will include:

- Gender

- Age Range

- Region

- Socio-economic group

- Ethnicity

- Specific measurement criteria - e.g. height range

Increasing the number of criteria and/or narrowing the range will necessarily reduce the number of subjects in the data subset and potentially weaken the statistical significance. The data subset should therefore be kept as large as possible without compromising the retailer's customer profile.

This is followed by a programme of measurement analysis designed to show:

- The overall size distribution of their customers

- The overall size range from smallest to largest

- Frequency distribution by size

- Market share by size

- The relationship between key measurements

- The effectiveness of their current size chart

This will enable decisions to be made on the upper and lower limits of the size chart and the intervals between the sizes. Size intervals can either be linear - where the interval is consistently the same from smallest size to largest - or non linear, where the interval between the sizes increases as sizes get bigger.

Typically a measurement analysis programme would include:

- Statistical tables giving the mean, median, min, max, standard deviation, $5^{\text {th }}$ and $95^{\text {th }}$ percentiles for each of the SizeUK measurements

- Frequency distribution charts, usually in $1 \mathrm{~cm}$ bands, for each of the key measurements e.g. bust girth, waist girth, hip girth and height. These show the size range from smallest to largest, the most frequently occurring sizes and the extent of the demand for small and large sizes. The results can be compared to current size charts to see how well the Retailers size current size chart covers their market. The charts can also be used to compare different age groups and regions.

- Measurement Correlation charts, plot 2 key measurements against each other for each of the subjects in the sample. They indicate how the concentration of subjects is distributed and show the relationship and extent of the correlation between the 2 measurements. These charts are useful for developing size charts - apart from showing the cluster concentrations they also help to delineate the overall size range.

- 3D measurement correlation charts also show the relationship between 2 measurements but provide more data on the most frequently occurring measurement combinations.

- Hit Rate Analysis calculates the percentage of the retailers target customer group that are satisfied by their current size chart.

- Size tables are based on one key measurement e.g. hip girth. For any given key measurement band the table shows the average of all the other SizeUK measurements. 
The output shows the overall size range, frequency distribution, measurement correlations and market share by size and provides the core information for the development of a new size chart.

\subsection{D Shape Analysis}

One of the major benefits of 3D body scanning is the wealth of 3D shape data that it generates. University College London (UCL) developed sophisticated 3D shape analysis software tools that enabled analysis of multiple 3D body scans for the first time. The shape analysis tools can generate $3 \mathrm{D}$ average body shapes from any subset of the data and also show the variation in shape across the sample. So, for example, a 3D average body shape can be generated from a specific set of subjects falling within a specific age and measurement range. Before SizeUK, retailers had no way of knowing the average body shape of their customers. Now they understand the shape of their customers as well as their measurements. This is clearly hugely valuable to an industry beset with problems of garment fit and size related returns. It also demonstrates that 3D shape analysis is the key to the successful implementation of size survey results.
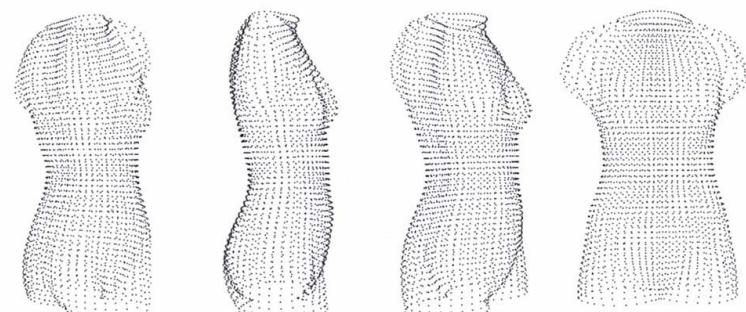

Fig.2 Four different views of an average female torso
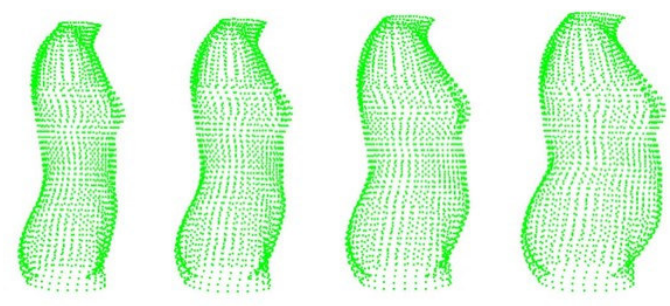

Fig.3 Average body shapes of underweight, normal, overweight and obese BMI body shapes

The output of 3D shape analysis can be shown as:

- $3 \mathrm{D}$ torso body shapes ( recently upgraded to full body analysis)

- $2 \mathrm{D}$ front, back and side profiles

- Horizontal slices at key measurement points

3D shape analysis has several applications which will be referred to in the rest of the paper:

- Size chart development

- Shape comparison

- 3D virtual mannequin development

- Physical mannequins development

- 3D pattern generation

\subsection{Size Chart Development}

Our approach to Size Chart Development is based on 3D shape data as opposed to linear measurements and traditional grade rules, and this differentiates the process. This is combined with a process of Size Chart Optimisation to maximise the percentage of the sample population covered by the chart.

The process starts with a programme of customised data analysis on a subset based on the retailer's target customer profile as described in 2.1. This is used to determine the upper and lower limits of the size chart and the interval between the sizes. The shape analysis software is then used to generate 3D average body shapes for each size.

The 3D average body shapes form the basis for the development of 3D virtual mannequins (avatars) one for every size in the range - starting with the main reference sizes and then filling in the intervening sizes through a process of interpolation. The resulting range of body shapes are aligned and interrelated. The size chart measurements are populated by extracting measurements from the 
3D virtual mannequins. Measurements are therefore the output of the process, not the input, and shape is the driver. Most importantly it means the measurements for each size in the chart relate directly to a body shape in a way that measurement based charts do not. Grade rules - the definition of the interval between sizes - are automatically generated from the 3D virtual bodies, demonstrating that the body does not grade in the same as traditional arithmetical grade rules. It is often impossible to create a body shape from a measurement based size chart without amending some of the measurements.

\subsection{Shape Comparison}

Individual 3D body shapes (3D body scans) can be compared to average body shapes to see how they differ. These can either be presented as simple 2D front, back and side profile overlays, 2D horizontal slice overlays or more advanced 3D comparisons. Shape comparison has a variety of applications. In the clothing industry they can be used to compare fit models to the 3D average body of the customers they are intended to represent. Fit models are rarely truly representative of the customer - both in terms of measurements, because they are often not measured accurately, or more importantly shape. The shape of a retailers fit models will often dictate the sizing and fit of the garments they produce, so if the model is not a representative shape it can have serious consequences in terms of limiting the number of potential customers that can fit their clothes.

3D body scanning and 3D shape comparison can be used to screen and select more representative fit models.

\subsection{Fit Mannequins}

Having established and understood the average body shape and size of their customers the Retailer now needs a means of implementing this into their product development process and across their manufacturing supply chain. Almost all high street retailers now source their manufacturing from low cost overseas markets. Many have also delegated responsibility for product development to the supplier. The supplier receives just a sketch and a measurement specification chart and submits a sample based on this information. The Retailer could have hundreds of suppliers all receiving the same information. On receipt of the sample the Retailer test fits the sample on their fit model. Not surprising it can take 3 or 4 iterations for the supplier to achieve the desired fit. Multiplied over hundreds of suppliers this is clearly an inefficient and costly process.

Fit mannequins, which have been historically used in the clothing industry for product development, provide Retailers with a means of establishing a fit standard across their supply chain where all their suppliers are developing product on the same body size and shape - the same shape that the retailer is using. They are also a practical tool that enables the retailer to implement the results of size survey data analysis right across their business. 3D shape analysis is again the key differentiator of our process.

The process starts with the development of a 3D virtual mannequin from a 3D average body shape (see 2.2.and 2.3). This ensures that the dummy is both a realistic representation of the human form as well as being truly representative of the clients target customer. It also removes subjectivity from the process. Amendments can be made to refine the body shape and ensure the development of a practical mannequin but the overall shape remains faithful to the original shape data.
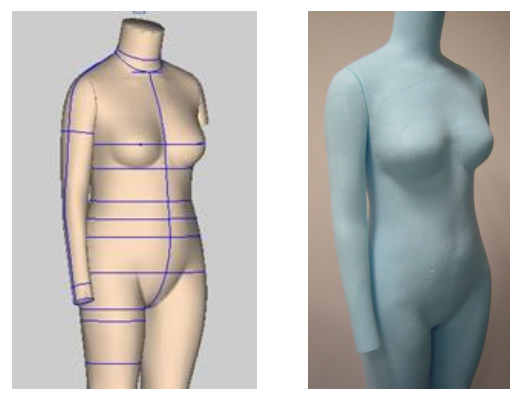

Fig.4 3D virtual mannequin (left) and prototype cut from polystyrene on a CNC rapid prototyping machine

It is also possible to compare these representative virtual body shapes with the 3D scans of fit models to determine their suitability.

The virtual mannequin is viewable as a fully rotatable $3 \mathrm{D}$ shape, but in order to realise and test the shape physically, we cut a hard copy prototype in polystyrene using a CNC rapid prototyping machine. 
The final linen covered mannequin is produced from moulds developed from the same 3D data to ensure accuracy and consistency

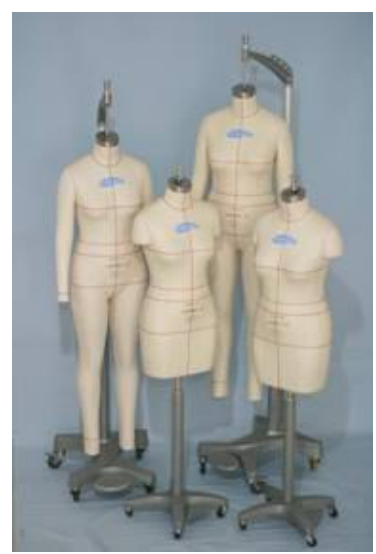

Fig.5 Finished linen covered mannequins mounted on adjustable metal stands

Sizemic has developed a programme of customised and generic fit mannequins based on SizeUK data. Customised mannequins are developed from SizeUK data based on the client's customer profile and sizing criteria. In collaboration with the SizeUK retailers Sizemic has developed a range of SizeUK Generic mannequins which are available for both genders in a range of sizes for 2 different age groups - younger and mature. The result is 2 distinctly different body shapes, noticeably characteristic of the age profile they represent.

The SizeUK mannequin programme has enabled retailers to implement the results of the survey across their supply chain whilst at the same time speeding up the product development process and significantly reducing cost. With greater reliance on international sourcing they have become an essential product development and quality communication tool.

\subsection{D Pattern Generation}

3D shape data can be used in a virtual environment as well as to create physical tools. The final stage in the commercialisation process is the development of pattern blocks based on the new body shapes. Pattern development is traditionally a 2D to 3D process. 'PPG', which stands for Parametric Pattern Generation software, is based on 3D body morphology and is a uniquely $3 \mathrm{D}$ to $2 \mathrm{D}$ process. This is an innovative and scientific approach to pattern development, the result of cumulative research over the past decade by our technology partner INSTITUT TPC (Alexandre Kung).

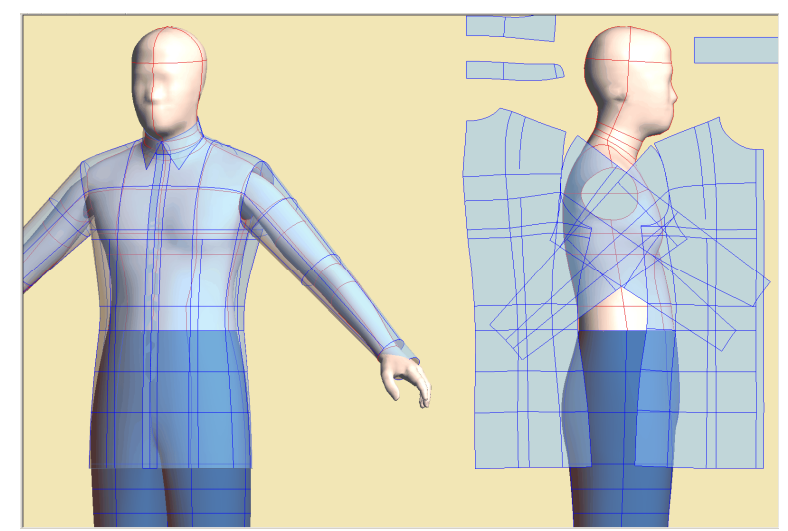

Fig.6 3D Parametric Pattern Generation Software (PPG)

PPG is a fully integrated sizing and garment fit solution. Garment structures are developed in a 3D environment on a range of virtual mannequins from which $2 \mathrm{D}$ patterns are generated in real time. Morphological grading ensures that the fit is replicated in all sizes and based on the 3D body shape of each size as opposed to traditional arithmetical grade rules. The result is greater accuracy of fit and better fitting garments in all sizes. Current product development practices tend to focus on getting the fit right in one sample size only (e.g. size 12) and rely on traditional grade rules for the sizing and fit of other sizes. This leads to inaccuracies and poor fit. As far as we are aware PPG is the only truly 3D pattern development software solution. 


\section{Commercial Benefits}

Commercialising and exploiting size survey data in this way enables retailers to enjoy several tangible benefits that improves the performance of their business, measureable through key performance indicators. The benefits can be divided into two distinct groups - those that are market oriented and impact on sales, margin, customer satisfaction and buying \& merchandising - and those that impact on the speed and cost of the product development process.

\subsection{Market Oriented, Sales Performance Benefits}

- Increased sales and reduced markdowns by maximising the percentage of their target customers that can fit their clothes.

- Increased customer satisfaction and loyalty through improvements to sizing and fit in all sizes.

- Producing more efficient size charts with more effective sizing increments.

- Understanding demand in specific size categories e.g. plus sizes \& petites and realising opportunities to develop new size related markets.

- Understanding market share by size, both nationally and regionally, exploited through improved buying ratios across sizes and more efficient allocation of stock to branches. This can help to reduce markdowns.

- Reduce size and fit related returns - this is especially relevant for home shopping retailers where returns rates are very high - but also applies to high street, bricks and mortar retailers too. Reducing returns, even by small amounts, has a significant impact on sales and margin.

\subsection{Product Development Benefits}

- Speeding up the product development process and reducing lead times by getting more samples right first time. Currently it can take 3 or 4 iterations of a sample before it is approved.

- Reducing the cost of product development and sampling - pattern development, sample making and shipping costs. These are incurred by the supplier but will be passed on to the retailer through higher prices.

- Reducing the number of fit sessions and fit model costs for the retailer. Typically a retailer will have 2 fit sessions per week lasting up to 7 hours. A fit model can cost around £20 per hour.

- Greater consistency of fit across the retailers supply chain - all suppliers fitting and developing product on same body size and shape, using the same block patterns

- Increases the level of quality control that a retailer imposes on the product development process.

- Improves communication with suppliers

\section{Conclusion}

Whilst the concentration has been on commercial applications of size survey data within the retail and fashion industry there are clearly many commercial applications in other industrial sector such as healthcare, sports \& fitness and transport to name just a few. There are also other applications within the clothing industry particularly relating to the use of $3 \mathrm{D}$ avatars for virtual design and $3 \mathrm{D}$ garment visualisation.

The prime focus of this paper has been to demonstrate how size survey data can be delivered in a form which enables Retailers in the clothing industry to implement the results and maximize the commercial benefits from investing in size survey data.

Sizing and fit remains a major issue for clothing retailers and a source of great frustration for many consumers. Retailers routinely spend large amounts on design, marketing and in-store merchandising to entice their customers to visit their stores and try on their clothes. Yet they spend relatively small amounts on ensuring that there is a reasonable chance that the clothes will actually fit. Given the investment up to this point it seems odd that they would not focus more on this critical factor in the purchasing decision. 3D body scanning and size survey data provides the basis for enabling Retailers to address this issue but if we are to succeed in convincing Retailers to invest in size survey data it is critically important that they are provided with the appropriate tools to exploit the data to the fullest extent possible.

Finally, the clothing industry has a tendency to be fixated with linear measurements and still works predominately in 2D. Yet the human body is 3 dimensional and comes in all sorts of shapes and sizes. 3D body scanning has spawned an array of new and innovative technologies that now enables the industry to work in a $3 \mathrm{D}$ environment and focus more on shape than linear measurements. Our task is to encourage clothing Retailers to embrace these new technologies and help them to realise the opportunities they open up. 